\title{
Características clínicas de las gestantes con hipertensión arterial crónica atendidas en un hospital general de Lima
}

\author{
Clinical characteristics of pregnants with chronic hypertension attended in a general hospital in Lima \\ Jordana Bravo ${ }^{1}$, Joana Sánchez ${ }^{1}$, Hector Sosa ${ }^{1}$, Jorge Díaz-Herrera ${ }^{2}$, Juan Miyahira ${ }^{3}$
}

\section{RESUMEN}

Objetivos: Describir las características epidemiológicas, clínicas y de laboratorio de las gestantes con hipertensión arterial crónica (HTAC), así como, conocer los resultados del producto de la gestación. Material y métodos: Estudio descriptivo, observacional, longitudinal, retrospectivo, tipo serie de casos. Se incluyeron pacientes gestantes con HTAC cuyo parto fue atendido en el Hospital Nacional Cayetano Heredia entre 2006 y 2012. Se utilizó estadística descriptiva, se determinaron frecuencias y porcentajes para las variables categóricas y medias y desviación estándar para las numéricas continuas. Resultados: Se incluyeron 41 gestantes con HTAC. El 56,1\% tenía 35 o más años, $68,3 \%$ presentó preeclampsia sobreagregada. La tasa de filtración glomerular estimada fue $<90 \mathrm{ml} / \mathrm{min}$ en $9,8 \%$, el $58,8 \%$ presentó proteinuria en 24 horas $>300 \mathrm{mg}$. El 73,2\% tuvo parto abdominal, 41,5\% de los recién nacidos fueron prematuros y el 39,1\% tuvo peso menor a 2500 g. Conclusiones: Las gestantes con HTAC presentan características clínicas y epidemiológicas similares a las descritas en otros estudios; con la diferencia que presentaron frecuencia alta de preeclampsia sobreagregada y complicaciones materno - perinatales.

PALABRAS CLAVE: Complicaciones del embarazo, hipertensión, resultado del embarazo. (Fuente: DeCS BIREME)

\section{SUMMARY}

Objectives: To describe epidemiological, clinical and laboratory characteristics of pregnants with chronic hypertension $(\mathrm{CH})$, as well as to investigate the outcome of their pregnancies. Methods: Case series that included pregnants with $\mathrm{CH}$ atttended at Hospital Nacional Cayetano Heredia from 2006 to 2012. Descriptive statistics were used for categorial and continuos variables. Results: A total of 41 pregnants with $\mathrm{CH}$ were included; $56 \%$ had at least 35 years of age; $68.3 \%$ presented associated pre-eclampsia; $9.8 \%$ had estimated glomerular filtration rates $<90$ $\mathrm{mil} / \mathrm{min}$, and $58.8 \%$ had proteinuria $>300 \mathrm{mg}$ measured in a 24 hour period. Abdominal delivery occured in $73.2 \%$; $41.5 \%$ of newborns were pre-term, and $39.1 \%$ had body weight less than $2500 \mathrm{~g}$. Conclusions: Pregnants with $\mathrm{CH}$ in this setting present with similar features as previously described in other studies, but higher rates of pre-eclampsia and mother-child complications were observed.

KEYWORDS: Pregnancy complications, hypertension, pregnancy outcome. (Source: MeSH NLM)

Egresado de la Facultad de Medicina Alberto Hurtado. Universidad Peruana Cayetano Heredia. Lima, Perú.

Médico Gineco-Obstetra. Doctor en Medicina. Médico asistente, Departamento de Ginecología, Obstetricia y Salud Reproductiva. Hospital Nacional Cayetano Heredia. Profesor Principal de la Facultad de Medicina Alberto Hurtado. Universidad Peruana Cayetano Heredia, Lima, Perú.

3 Médico Nefrólogo. Maestro en Medicina. Médico asistente, Servicio de Nefrología. Departamento de Medicina, Hospital Nacional Cayetano Heredia. Profesor Principal de la Facultad de Medicina Alberto Hurtado. Universidad Peruana Cayetano Heredia. Lima, Perú. 


\section{INTRODUCCIÓN}

La hipertensión es una de las complicaciones más comunes de la gestación, observándose que $1 \mathrm{a}$ $5 \%$ de las gestantes presentan hipertensión arterial crónica (HTAC) y su prevalencia está en aumento. Este incremento se atribuye a la mayor frecuencia de obesidad y a la edad materna avanzada en la que es más común la HTAC (1).

Durante la gestación, la mujer con HTAC y su producto están sometidos a diversos riesgos, sea por la enfermedad o por patología asociada. Aumenta el riesgo de pre eclampsia; un estudio realizado en gestantes con HTAC se encontró que esta condición sumada a una historia de preeclampsia en una gestación previa, o HTAC de por lo menos cuatro años de duración o una presión arterial diastólica de 100 $\mathrm{mm} \mathrm{Hg}$ o más al inicio de la gestación, se asociaron a una mayor frecuencia de preeclampsia sobreagregada (2). El desprendimiento prematuro de placenta (DPP) es otra complicación en gestantes con HTAC, su incidencia es $1,5 \%$ pero aumenta a $3 \%$ en las que desarrollaron preeclampsia sobreagregada (3).

Dentro de las complicaciones en el producto de la gestación en gestantes con HTAC se ha descrito parto pretérmino, recién nacido pequeño para la edad gestacional (PEG), mayor admisión a cuidados intensivos de neonatología y un incremento en la mortalidad perinatal; que podrían tener relación con la presencia de proteinuria al inicio de la gestación $(3,4)$. Sin embargo, las investigaciones realizadas no son suficientes para extrapolar dichos resultados a nuestra población.

El objetivo del estudio fue describir las características epidemiológicas, clínicas y obstétricas de las gestantes con HTAC, así como las características del producto de la gestación.

\section{MATERIAL Y MÉTODOS}

Estudio descriptivo, longitudinal, observacional, retrospectivo, tipo serie de casos. Se incluyeron gestantes con diagnóstico de HTAC registrado en la historia clínica, con o sin patología asociada cuyo parto fue atendido en el Hospital Nacional Cayetano Heredia entre 2006 y 2012.

HTAC fue definido como hipertensión presente y observable antes del embarazo o que se diagnosticó antes de la $20^{\circ}$ semana de gestación o que se diagnosticó por primera vez durante la gestación y no se resolvió en el post parto. Hipertensión fue definida como la presión sistólica $\geq 140 \mathrm{~mm} \mathrm{Hg}$ o diastólica $\geq$ $90 \mathrm{~mm} \mathrm{Hg}(4)$.

La búsqueda de las pacientes se realizó en la base de datos del Carnet Perinatal (CLAP), del cuaderno de procedimientos del test no estresante (NST) y del test estresante (CST) del Departamento de Ginecología y Obstetricia y de un registro propio de la consulta externa de uno de los investigadores. Se encontraron 68 pacientes de la base del CLAP, 2 pacientes de la segunda fuente y 7 pacientes de la última, haciendo en total 77 pacientes. Se excluyeron 25 que no tenían diagnóstico de HTAC, 9 pacientes por no encontrar la historia clínica y 2 pacientes con historia clínica incompleta. Finalmente se incluyeron 41 pacientes.

La recopilación de los datos se realizó en una ficha elaborada por los investigadores. Se registraron los siguientes datos: edad, número de gestaciones, peso, talla, número de controles prenatal, comorbilidad, tiempo de enfermedad hipertensiva, exámenes de laboratorio (hematocrito, plaquetas, creatinina, proteinuria), presencia de preeclampsia o síndrome HELLP, presencia de desprendimiento prematuro de placenta, tipo de parto y medicación recibida; edad gestacional al momento del parto, peso del neonato, APGAR al minuto. Se consideró proteinuria cuando era mayor de $300 \mathrm{mg}$ en 24 horas.

Los datos obtenidos fueron procesados en el programa SPSS versión 19 previa codificación. Se utilizó estadística descriptiva, se determinaron frecuencias y porcentajes para las variables categóricas y medias y desviación estándar para las variables numéricas continuas. Se utilizó Chi cuadrado para el contraste de proporciones y se consideró significativo un $\mathrm{p}<0,05$.

El estudio fue revisado y aprobado por el Comité de Ética de la Universidad Peruana Cayetano Heredia.

\section{RESULTADOS}

La edad promedio fue 35,8 $\pm 5,13$ años y el IMC promedio $28,4 \pm 5,5 \mathrm{Kg} / \mathrm{m} 2$. Las características epidemiológicas de las pacientes se muestran en la Tabla 1. El 70,7\% era multigesta y alrededor de la tercera parte era obesa, pero casi la mitad tenía sobrepeso. Siete pacientes tenían enfermedad renal crónica, dos de ellas recibían hemodiálisis.

Las características clínicas se observan en la tabla 
2. El tiempo de enfermedad promedio de la población fue $63,7 \pm 69,8$ meses (5,3 años). El hematocrito promedio al final de la gestación fue $33,3 \pm 5,2 \%$ y en ningún caso hubo hemoconcentración. La proteinuria promedio fue mayor a un gramo y fue $>300 \mathrm{mg}$ en 24 horas en más de la mita d de las pacientes y más del $90 \%$ tenía Tasa de filtración glomerular calculada $>90$ $\mathrm{ml} / \mathrm{min}$

Un número reducido de pacientes tuvo evaluación para daño de órgano blanco, se encontró retinopatía en $8 / 15(53,3 \%)$, cardiopatía en 8/11 (72,7\%) y nefropatía en $1 / 8(12,5 \%)$.

Se encontró que la presión arterial sistólica (PAS), diastólica (PAD) y media (PAM) fueron mayores en el tercer trimestre, comparados con el segundo y primer trimestre (Gráfico 1).

En el gráfico 2 se muestran las medidas inicial y final de la creatinina sérica en 15 pacientes y

Tabla 1. Características epidemiológicas de las gestantes con Hipertensión arterial crónica.

\begin{tabular}{lrr}
\hline & n & \% \\
\hline Edad & & \\
$\quad<35$ años & 18 & 43,9 \\
$\quad \geq 35$ años & 23 & 56,1 \\
IMC & & \\
$\quad<25 \mathrm{Kg} / \mathrm{m}^{2}$ & 9 & 22,0 \\
$25-29 \mathrm{Kg} / \mathrm{m}^{2}$ & 19 & 46,3 \\
$\quad \geq 30 \mathrm{Kg} / \mathrm{m}^{2}$ & 13 & 31,7 \\
Número de gestaciones & & \\
$\quad$ Primigesta & 3 & 7,3 \\
$\quad$ Segundigesta & 5 & 12,2 \\
$\quad$ Tercigesta & 4 & 9,8 \\
$\quad$ Multigesta $(\geq 4)$ & 29 & 70,7 \\
Comorbilidades & & \\
$\quad$ Ninguna & 17 & 41,5 \\
$\quad$ Diabetes & 2 & 4,9 \\
Obesidad & 13 & 31,7 \\
$\quad$ Enfermedad renal crónica & 7 & 17,1 \\
$\quad$ Otros * & 7 & 17,1 \\
Control Prenatal & & \\
$\quad \geq 4$ controles & 27 & 65,9 \\
$\quad<4$ controles & 14 & 34,1 \\
\hline
\end{tabular}

* Enfermedad tiroidea (4 pacientes), dislipidemia (2 pacientes), cardiopatía congénita (1 paciente)
Tabla 2: Características clínicas y de laboratorio de las gestantes con Hipertensión arterial crónica.

\begin{tabular}{lcc}
\hline & $\mathbf{n}$ & $\mathbf{\%}$ \\
\hline $\begin{array}{l}\text { Tiempo de enfermedad } \\
\quad<4 \text { años }\end{array}$ & 23 & 56,1 \\
$\quad \geq 4$ años & 18 & 43,9 \\
$\quad$ Hematocrito & & \\
$\quad \geq 33 \%$ & 26 & 63,4 \\
$\quad<33 \%$ & 15 & 36,6 \\
Plaquetas & & \\
$\quad \geq 150000 / \mathrm{mm}^{3}$ & 39 & 95,1 \\
$<150000 / \mathrm{mm}^{3}$ & 2 & 4,9
\end{tabular}

Tasa de Filtración Glomerular estimada

$\begin{array}{lcc}\geq 90 \mathrm{ml} / \mathrm{min} & 37 & 90,2 \\ <90 \mathrm{ml} / \mathrm{min} & 4 & 9,8 \\ \text { roteinuria (> } 300 \mathbf{~ m g / 2 4} \text { horas) } & & \\ \text { No } & 14 / 34 & 41,2 \\ \text { Sí } & 20 / 34 & 58,8\end{array}$

Electrocardiograma

$\begin{array}{lcc}\text { Normal } & 34 & 82,9 \\ \text { Arritmia } & 3 & 7,3 \\ \text { Hipertrofia ventricular izquierda } & 4 & 9,8\end{array}$

Daño de Órgano Blanco

\begin{tabular}{lcc} 
Retinopatía & $11 / 15$ & 56,3 \\
Accidente Cerebrovascular & 0 & 0,0 \\
Cardiopatía & $8 / 11$ & 72,7 \\
Nefropatía & $1 / 8$ & 12,5 \\
\hline Tasa de filtración glomerular calculada por la fórmula de \\
Cockroft-Gault.
\end{tabular}

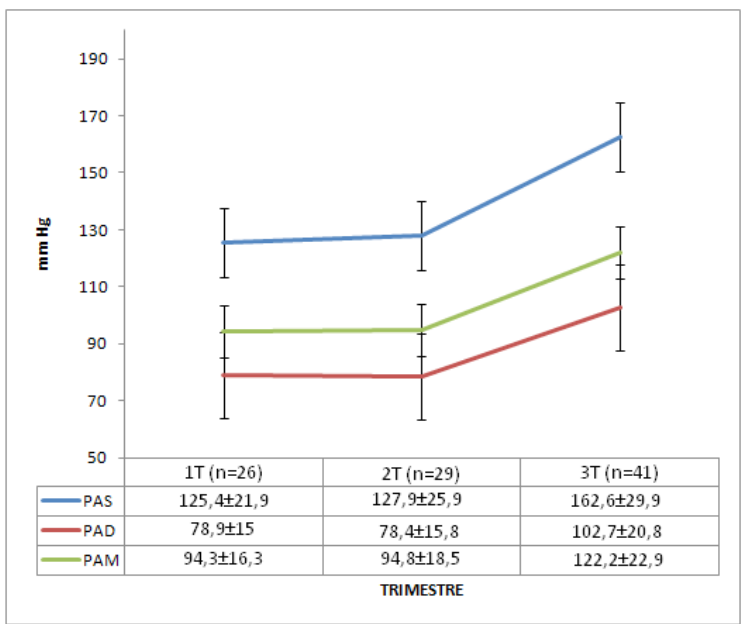

Gráfico 1. Presión arterial sistólica (PAS), diastólica (PAD) y media (PAM) promedio en cada trimestre en gestantes con hipertensión arterial crónica. 


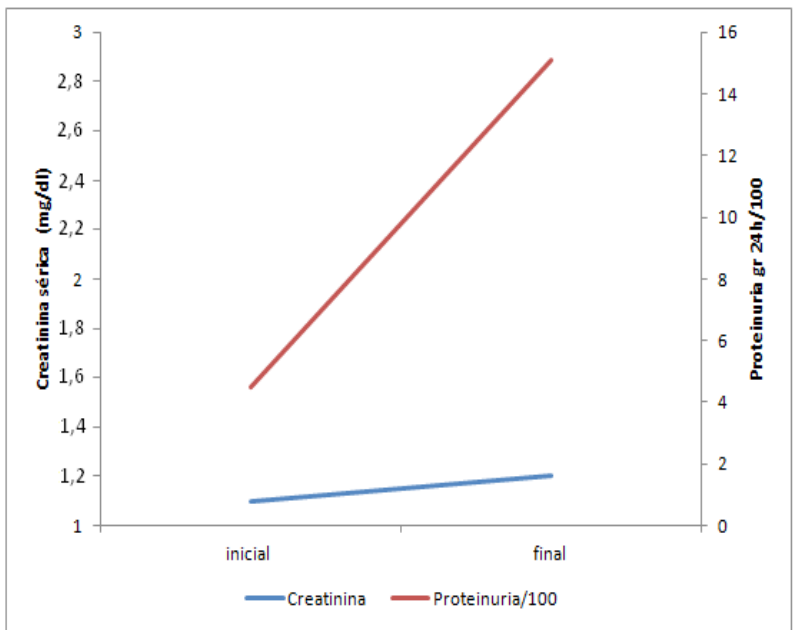

Gráfico 2. Cambios en la creatinina sérica $(n=15)$ y proteinuria promedio $(\mathrm{n}=17)$ durante la gestación en pacientes con Hipertensión arterial crónica.

Tabla 3. Complicaciones maternas de las gestantes con Hipertensión arterial crónica.

\begin{tabular}{lrc}
\hline & n & \% \\
\hline Diagnóstico & 13 & 31,7 \\
HTAC & 6 & 14,6 \\
HTAC con Preeclampsia leve & 18 & 43,9 \\
HTAC con Preeclampsia severa & 4 & 9,8 \\
$\quad$ HTAC con Preeclampsia severa & & \\
y Síndrome HELLP* & 2 & 4,9 \\
$\begin{array}{l}\text { Desprendimiento Prematuro de } \\
\text { Placenta }\end{array}$ & & \\
Tipo de parto & 11 & 26,8 \\
$\quad$ Vaginal & 30 & 73,2 \\
$\quad$ Cesárea &
\end{tabular}

*HELLP: (Siglas en inglés) (H) Hemólisis, (EL) enzimas hepáticas elevadas - (LP) conteo bajo de plaquetas.

proteinuria en 17 pacientes. Se observó un incremento significativo en la proteinuria $(\mathrm{p}=0,02)$, mientras que la creatinina sérica permaneció sin cambios $(\mathrm{p}=0,5)$.

El $68,3 \%$ presentó preeclampsia sobreagregada. La mayoría fue severa (Tabla 3). Alrededor de las tres cuartas partes requirió cesárea. No se reportaron muertes maternas.

Las características del recién nacido se muestran en la tabla 4. El $41,5 \%$ de las pacientes tuvieron parto pretérmino, de ellas $58,8 \%$ tenían entre 34 y 36 semanas y $41,2 \%$ menos de 34 semanas. Alrededor de la cuarta parte de los neonatos fueron pequeños para
Tabla 4. Características del producto de la gestación en gestantes con Hipertensión arterial crónica $(n=41)$.

\begin{tabular}{lcc}
\hline & $\mathbf{n}$ & \% \\
\hline $\begin{array}{l}\text { Edad Gestacional } \\
\quad \text { A término }\end{array}$ & 24 & 58,5 \\
$\quad$ Pretérmino & 17 & 41,5 \\
Peso al nacer $(\mathbf{n = 3 9 )}$ & & \\
$\quad$ AEG & 30 & 76,9 \\
$\quad$ PEG & 9 & 23,1 \\
APGAR al minuto (n=37) & & \\
$\quad \geq 8$ & 31 & 83,8 \\
$\quad<8$ & 6 & 16,2 \\
Muerte Fetal & 2 & 4,9 \\
Líquido meconial & 1 & 2,4 \\
\hline
\end{tabular}

AEG: Adecuado para edad gestacional; PEG: pequeño para edad gestacional

Tabla 5. Tratamiento antihipertensivo requerido por las pacientes con Hipertensión arterial crónica durante la gestación.

\begin{tabular}{lrr}
\hline & n & \% \\
\hline $\begin{array}{l}\text { Número de antihipertensivos } \\
\text { recibidos } \\
\quad \text { Ninguno }\end{array}$ & 4 & 9,8 \\
1 medicamento & 16 & 39,0 \\
2 medicamentos & 15 & 36,6 \\
3 o más medicamentos & 6 & 14,6 \\
Tiempo de tratamiento & & \\
$\quad$ Todo el embarazo & 20 & 48,8 \\
$\quad$ Periparto & 21 & 51,2 \\
\hline
\end{tabular}

la edad gestacional (PEG). Ocurrieron dos muertes fetales en pacientes con edad materna avanzada.

Alrededor de la mitad de las pacientes requirió dos o más antihipertensivos (Tabla 5). Los antihipertensivos utilizados con más frecuencia fueron alfametildopa, amlodipino y nifedipino.

No se encontró asociación significativa entre la presencia de preeclampsia y el número de controles del CPN $(p=0,08)$, peso para la edad gestacional $(\mathrm{p}=0,5)$, tiempo de enfermedad $(\mathrm{p}=0,57)$, o número de gestaciones $(\mathrm{p}=0,63)$. 


\section{DISCUSIÓN}

En nuestra serie la edad promedio de las pacientes fue mayor de 35 años, mayor que la encontrada en otro estudio (33 $\pm 5,8$ años) (5). Esto se puede explicar por la tendencia en los últimos años, a diferir los embarazos.

Alrededor de la mitad de las pacientes presentaron sobrepeso u obesidad, hallazgo similar al encontrado en otros estudios $(5,6)$. La mayoría de pacientes fue multigesta; un estudio en México tuvo un hallazgo similar (5), sin embargo, otro estudio encontró que el $42,2 \%$ de las pacientes eran nulíparas (7).

El tiempo de enfermedad promedio de nuestras pacientes fue mayor de 5 años. En el estudio mexicano la edad promedio fue 3,4 $\pm 2,4$ años (5). Alrededor de la mitad tenía un tiempo de enfermedad mayor a 4 años, población en la que se ha descrito mayor riesgo de desarrollar preeclampsia sobreagregada (2).

En nuestro estudio, $58,8 \%$ de pacientes presentó proteinuria, probablemente asociada a la alta tasa de preeclampsia sobreagregada encontrada en nuestra serie. Solo alrededor de 10\% tuvo tasa de filtración glomerular (TFG) disminuida $(<90 \mathrm{ml} / \mathrm{min})$. Un estudio que comparó la TFG al inicio y al final de la gestación mostró que las pacientes con proteinuria basal $>1$ g y una $\mathrm{TFG}<40 \mathrm{ml} / \mathrm{min}$, presentaron una mayor pérdida de la TFG (8).

No se pudo evaluar el daño de órgano blanco al no contar con una muestra adecuada de pacientes. Idealmente, las evaluaciones de la función renal, de la retina y del compromiso cardiovascular en una paciente con HTAC, deberían realizarse antes o durante las primeras semanas de gestación, para identificar el daño de órgano blanco (alto riesgo) y realizar una evaluación seriada de proteinuria en 24 horas, al menos una vez por trimestre $(6,9,10)$.

Se encontró una tendencia al aumento de la presión arterial con el tiempo de gestación, con mayor aumento en el tercer trimestre; inclusive en el primer y segundo trimestre cuando normalmente hay disminución de la presión arterial debido a una reducción en la resistencia vascular sistémica, la PAM de nuestras pacientes se encontró en un valor por encima de lo normal en las etapas tempranas de la gestación (11).

La complicación más frecuente fue la preeclampsia sobreagregada siendo la frecuencia encontrada mayor a la de otros estudios los que reportan entre 4,7 y $52 \%(5,12)$. Una de las explicaciones sería la mayor edad de nuestras pacientes; otra por variables socio-demográficas como el nivel socio-económico. En un estudio realizado en México se encontró que $73,9 \%$ de las pacientes con preeclampsia tenían nivel socioeconómico bajo (13).

Casi las $3 / 4$ partes de las pacientes tuvieron parto abdominal, cifra mayor al $60 \%$ descrito en la literatura; lo que podría estar asociado a la frecuencia de preeclampsia (10). En un estudio peruano la frecuencia de cesárea en mujeres con preeclampsia fue $69,3 \%$ cifra similar a la encontrada en nuestra serie (14).

La frecuencia de parto prematuro encontrada en nuestro estudio fue similar al de otros estudios (25$40 \%$ ) (10), pero la proporción de recién nacidos con peso bajo al nacer, es superior a las de otros estudios $(5,9)$. Las pacientes con enfermedad renal crónica terminal contribuyen a esta cifra en nuestra serie, coincidiendo con otros estudios (15). La frecuencia de muerte fetal $(4,9 \%)$ fue ligeramente mayor a la reportada en otros estudios $(0,9$ - 3\%) $(5,6)$. No se estudió la mortalidad neonatal que puede dar otra dimensión al problema (16).

La mayoría de pacientes recibió metildopa, que es el antihipertensivo de primera línea recomendado en guías clínicas internacionales y el de mayor seguridad para el feto $(5,10,14)$. Alrededor de la mitad de los pacientes requirió 2 a 3 antihipertensivos, lo que muestra la severidad de la hipertensión arterial; otro estudio encontró $70 \%$ (3). La mayoría de las pacientes recibió antihipertensivos en el periparto.

Las gestantes quienes no utilizaron medicamentos antihipertensivos, tuvieron productos con peso normal al nacer. Varios estudios incluyendo un metaanálisis describen un posible efecto adverso en los neonatos de gestantes con HTAC leve que usan antihipertensivos $(10,17,18)$.

Un estudio encontró que el 75,3\% de PEG provenían de gestantes con preeclampsia (7); en nuestro estudio no se encontró asociación entre preeclampsia y bajo peso al nacer. Tampoco se encontró asociación entre preeclampsia y tiempo de diagnóstico de HTAC $\geq 4$ años, como en un estudio previo (2).

Las limitaciones del estudio son las derivadas de un estudio retrospectivo. Hubo un inadecuado registro de diagnósticos de las pacientes y durante la recolección 
de datos se observó un inadecuado seguimiento de las pacientes con HTAC, por lo que el pequeño número de pacientes, no permitió encontrar asociaciones que podrían existir.

En conclusión, hemos observado que las gestantes con HTAC presentan características clínicas y epidemiológicas similares a las descritas en otros estudios; con la diferencia que presentaron frecuencia alta de preeclampsia sobreagregada, elevada tasa de cesárea y complicaciones materno - perinatales. Al tratarse de una serie de casos sabemos que las conclusiones obtenidas no son definitivas, pero los datos obtenidos permitirán estudios posteriores.

\section{Declaración de financiamiento y de conflictos de interés:}

El estudio fue financiado por los investigadores, quienes declaran no tener conflictos de intereses.

\section{Correspondencia:}

Joana Sánchez

Correo electrónico: joana.sanchez@upch.pe

\section{REFERENCIAS BIBLIOGRÁFICAS}

1. Small MJ, Hayslett JP. Chronic hypertension in pregnancy. En: Funai EF, Evans MI, Lockwood ChJ, editors. High risk obstetrics. Philadelphia: Mosby Elsevier; 2008.p. 241-56.

2. Sibai BM, Lindheimer $M$, Hauth $J$, et al. Risk factors for preeclampsia, abruption placentae, and adverse neonatal outcomes among women with chronic hypertension. N Engl J Med. 1998; 339:667671.

3. Seely EW, Ecker J. Chronic hypertension in pregnancy. N Engl J Med. 2011; 365:439-446.

4. Report of the National High Blood Pressure Education Program Working Group on High Blood Pressure in Pregnancy. Am J Obstet Gynecol. 2000; 138:S1-S22.

5. Iñigo CA, Torres LG, Vargas A, et al. Hipertensión arterial crónica en 110 mujeres embarazadas. Ginecol Obstet Mex. 2008; 76(4):202-210.

6. Cifuentes R. Ginecología y obstetricia basadas en las nuevas evidencias. 2da Ed. Bogotá: Distribuna Editorial Médica; 2008. p. 279 - 84.
7. Allen V, Joseph K, Murphy K, et al. The effect of hypertensive disorders in pregnancy on small for gestational age and stillbirth: a population based study. BMC Pregnancy and Childbirth. 2004; 4:17.

8. Imbasciati E, Gregorini G, Cabiddu G, et al. Pregnancy in CKD Stages 3 to 5: Fetal and Maternal Outcomes. Am J Kidney Dis. 2007; 49: 753- 762.

9. Sibai BM. Chronic hypertension. En: Queenan J, Hobbins J, Spong CY, editors. Protocols for High Risk Pregnancies. New Jersey: Wiley-Blackwell; 2010. p. 264-58.

10. American College of Obstetricians and Gynecologists. Chronic hypertension in pregnancy. ACOG Practice Bulletin N ${ }^{\circ}$ 125. Obstet Gynecol. 2012; 119:396-407.

11. Grindheim G, Estensen ME, Langesaeter E. Changes in blood pressure during healthy pregnancy: a longitudinal cohort study. J Hypertens. 2012; 30(2) 342-50.

12. Powrie R. A 30 year old woman with chronic hypertension trying to conceive. JAMA. 2007; 298(13):1548-58.

13. Morgan F, Calderón S, Martínez J, et al. Factores de riesgo asociados con preeclampsia: estudio de casos y controles. Ginecol Obstet Mex. 2010; 78(3):153-159.

14. Salviz M. Factores de riesgo para preeclampsiaeclampsia. Tesis de Bachiller en Medicina. Lima, Perú. Universidad Peruana Cayetano Heredia, 1994. $11 \mathrm{pp}$.

15. Nevis IF, Reitsma A, Dominic A, et al. Pregnancy outcomes in women with chronic kidney disease: a systematic review. Clin J AM Soc Nephrol. 2011; 6(11):2587-98.

16. Vigil-De Gracia P, Lasso M, Montufar-Rueda C. Perinatal outcome in woman with severe chronic hypertension during the second half of pregnancy. Int J Gynaecol Obstet. 2004; 85:139-44.

17. Von Dadelszen P, Ornstein MP, Bull SB, et al. Fall in mean arterial pressure and fetal growth restriction in pregnancy hypertension: a meta-analysis. Lancet. 2000; 355:87-92.

18. Su CY, Lin HC, Cheng HC, Yen AM, Chen YH, Kao S. Pregnancy outcomes of anti-hypertensives for women with chronic hypertension: a populationbased study. PLoS One. 2013; 8(2):1-9.

Recibido: 20/05/2013

Aceptado: 25/11/2013 